\title{
Influence of turbulence on the shape of a spectral line: the analytical approach
}

\author{
N. A. Silant'ev ${ }^{1,2}$, E. E. Lekht ${ }^{1}$, J. E. Mendoza-Torres ${ }^{1}$ \\ and G. M. Rudnitskij ${ }^{3}$ \\ ${ }^{1}$ Instituto Nacional de Astrofísica, Óptica y Electrónica, Luis Enrique Erro No. 1, \\ Apdo Postal 51 y 216, 72840 Tonantzintla, Puebla, México \\ email: silant@inaoep.mx \\ ${ }^{2}$ Main Astronomical Observatory, Russian Academy of Sciences, \\ Pulkovo, St.-Petersburg, 196140 Russia \\ ${ }^{3}$ Sternberg Astronomical Institute, Moscow State University, \\ 13 Universitetskij prospekt, Moscow, 119992 Russia \\ email: gmr@sai.msu.ru
}

\begin{abstract}
We consider the propagation of spectral-line radiation in a correlated turbulent atmosphere. The ensembles of turbulent velocities $\mathbf{u}(\mathbf{r}, t)$ and optical depths, $\tau_{\nu}$, are assumed to be Gaussian. We investigate the explicit analytical solution of the stochastic radiative transfer equation for the intensity $I_{\nu}$ of radiation. The scattering term is not taken into account. It is shown that, in addition to the usual Doppler broadening of the spectral line, correlated turbulent motions of atoms and molecules give rise to considerable changes in the shape of a spectral line. We find that the mean intensity $I_{\nu}^{(0)}\left(I_{\nu}=I_{\nu}^{(0)}+I_{\nu}^{\prime},\left\langle I_{\nu}^{\prime}\right\rangle=0\right)$ obeys the usual radiative transfer equation with renormalized extinction factor $\alpha_{\nu}^{\text {eff }}$ if the correlation length $R_{0}$ of the turbulence is small as compared to a photon free path. A simple analytical expression for $\alpha_{\nu}^{\text {eff }}$ is given. This expression integrally depends on the two-point correlation function of the turbulent velocity field.
\end{abstract}

Keywords. turbulence, molecular processes, radio lines: ISM, radio lines: stars, masers: circumstellar matter

In a turbulent layer the effective absorption coefficient $\alpha_{\nu}^{\text {eff }}$ does not coincide with the mean absorption coefficient $\alpha_{\nu}^{(0)} \equiv\left\langle\alpha_{\nu}\right\rangle$. The transition $\alpha_{\nu}^{\text {eff }} \rightarrow \alpha_{\nu}^{(0)}$ takes place if the optical depth at the line center $\tau_{1}$ of correlation length $R_{0}$ tends to zero (the case of short-correlated turbulence), or if the characteristic thermal velocity $u_{\mathrm{Th}}$ is equal to or greater than characteristic turbulent velocity $u_{k}$ (parameter $\xi=u_{\mathrm{Th}} / u_{k} \geqslant 1$ ).

It is found that $\alpha_{\nu}^{\mathrm{eff}} \leqslant \alpha_{\nu}^{(0)}$. Statistically, a turbulent layer with a finite correlation length is more transparent than a layer of short-correlated turbulence for all the frequencies of a spectral line. It can be estimated even from the simple example of the averaging of intensities corresponding to two realizations with optical lengths $\tau_{\nu}^{(0)}+\tau_{\nu}^{\prime}$ and $\tau_{\nu}^{(0)}-\tau_{\nu}^{\prime}$.

The averaged spectral line intensity $I_{\nu}^{(0)}$ is narrower than that described by the radiative transfer equation with an averaged extinction factor $\alpha_{\nu}^{(0)}$ (see Fig. 1). This means that turbulence with a finite correlation length diminishes the equivalent width of an absorption line as compared with short-correlated turbulence.

The distortion of the shape increases with increasing optical depth $\tau_{0}$ of the turbulent layer at the line center. An increase in the parameter $\tau_{1}$ and decrease in $\xi$ also give rise to an increase in the distortion.

If the turbulent layer contains many $\left(\tau_{0} / \tau_{1} \geqslant 1\right)$ turbulent cells (rotating curls), then the effects of finite correlation length turbulence disappear. If the number of turbulent 


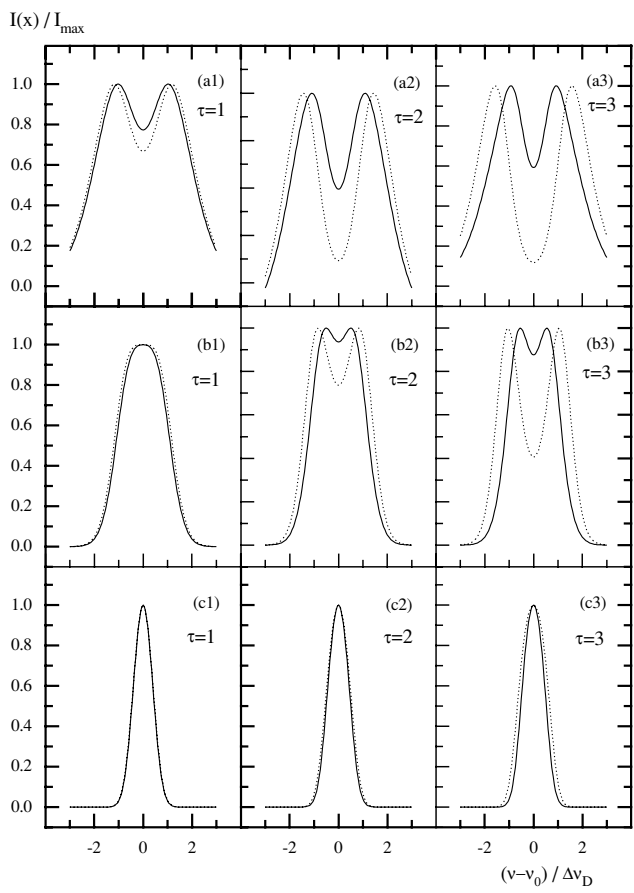

Figure 1. Normalized shape $I_{\nu}(x) / I_{\nu \max }$ of the maser line with a Gaussian initial profile after the passage of a turbulent cloud (solid lines). The dotted lines correspond to the usual model of short-correlated turbulence. The cases (an), (bn) and (cn) represent $\Delta \nu_{D} / \Delta \nu_{S}=0.5,1$ and 2 , respectively; $\Delta \nu_{D}$ and $\Delta \nu_{S}$ are the Doppler widths in the cloud and maser source. The optical depth of the cloud $\tau$ at the line center takes the values 1,2 and 3. Turbulence correlation length $R_{0}$ is taken to be one-fifth of the layer's depth, and parameter $\xi=u_{\mathrm{Th}} / u_{k}=0.1 ; u_{\mathrm{Th}}$ and $u_{k}$-values are characteristic thermal and turbulent velocities in the cloud.

cells is not large, but $u_{T h} \geqslant u_{k}$, the finite correlation length effects also tends to disappear. In the first case the situation looks like a cloud of many chaotically moving absorbing atoms. The second case corresponds to a very small shift of the center of the $\alpha_{\nu}$ - profile for either correlated or uncorrelated turbulence.

These effects were mentioned earlier in various papers as the results of purely numerical calculations (see, for example, Gail et al. 1974, Levshakov et al. 1997, Magnan et al. 1976), without a simple physical explanation. The simple analytical formulae derived in our paper (Silant'ev et al. 2006) allow us to understand the expected effects of turbulence without complicated numerical calculations.

In our paper (Silant'ev et al. 2006) we discuss the problem of how to obtain the main turbulent parameters from the analysis of the shape of an absorption line or maser line propagating through a turbulent interstellar cloud. It should be emphasized that in the case of finite correlation length turbulence one can estimate separately the characteristic thermal velocity $u_{T h}$ and turbulent velocity $u_{k}$. This is impossible if one uses the shortcorrelated model of turbulence.

As an illustrative example, we have estimated the possible parameters of the $\mathrm{H}_{2} \mathrm{O}$ maser source in S252A (Berulis et al. 1996). We hope that the obtained analytical formulae will be useful for a detailed explanation of various observational data.

\section{References}

Berulis, I. I., Lekht, E. E., Mendoza-Torres, J. E., 1996, Astron. Rep. 40, 329

Gail, H.-P., Hundt, E., Kegel, W. H., et al. 1974, Astron. Astrophys. , 32, 65

Levshakov, S. A., \& Kegel, W. H. 1997, MNRAS 288, 787

Magnan, C. 1976, J. Quant. Spectrosc. Radiat. Transfer 16, 281

Silant'ev, N. A., Lekht, E. E., Mendoza-Torres, J. E., Rudnitskij, G. M., 2006, Astron. and Astrophys. 453, 989 